\title{
Análisis de las inversiones del intrón 1 y 22 y secuenciación del gen $F 8$ para el diagnóstico genético-molecular de hemofilia A en Chile
}

\author{
HELENA POGGI $^{1, \mathrm{a}}$, JOSEFINA HONORATO ${ }^{\mathrm{a}}$, ELIANA ROMEO ${ }^{1, \mathrm{~b}}$, \\ PAMELA ZÚNIIGA ${ }^{2}$, TERESA QUIROGA ${ }^{1}$, MARCELA LAGOS ${ }^{1}$

\section{Introns 1 and 22 inversions and $F 8$ gene sequencing for molecular diagnosis of hemophilia A in Chile}

\begin{abstract}
Background: Hemophilia $A$ is an inherited disorder caused by alterations in factor VIII gene (F8) located on the X-chromosome, the intron 22 inversion being the most common mutation. The rest are predominantly point mutations distributed along this large gene of 26 exons. Aim: To implement a molecular diagnostic test to detect mutations in the F8 gene in Chilean patients with Hemophilia A. Material and Methods: To validate the testing methods, we analyzed samples with intron 22 and intron 1 inversion, and with point mutations previously studied, as well as one subject without Hemophilia. We also studied unrelated Chilean patients with Hemophilia $A$ and their female relatives for carrier testing. Intron 22 and intron 1 inversions were studied by long distance polymerase chain reaction (PCR) and point mutations by sequencing the coding and promoter regions of the F8 gene. Results: The results obtained in all samples used for validation were concordant with those obtained previously. In the Chilean patients, the intron 22 inversion and point mutations previously described were observed. In 6 out of 9 patients with mild Hemophilia A we found the same mutation (Arg2159Cys) in exon 23, which has been described as prevalent in mild Hemophilia A. Conclusions: The analysis of intron 22 and intron 1 inversions, as well as of point mutations in the F8 gene will help us to confirm the diagnosis in patients with severe, moderate and mild Hemophilia A, and also it will allow us to perform carrier testing and to provide better genetic counseling.
\end{abstract}

(Rev Med Chile 2011; 139: 189-196).

Key words: Hemophilia A; Genetic counseling; Genetic testing.

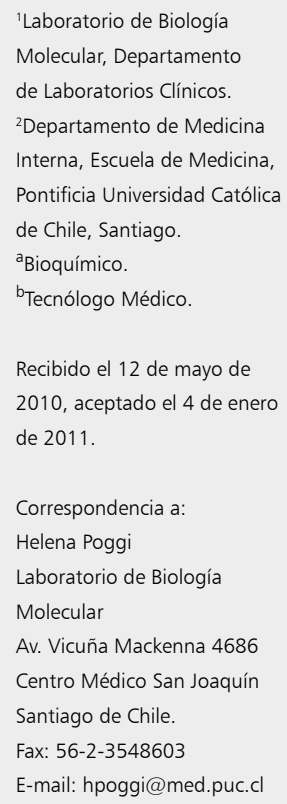

L a hemofilia A es una enfermedad hereditaria, ligada al cromosoma $\mathrm{X}$, que se caracteriza por un defecto cuantitativo o funcional del factor VIII de la coagulación sanguínea y afecta 1 por cada 5.000-10.000 hombres. De acuerdo a la guía clínica para hemofilia del Ministerio de Salud del año 2007, en Chile se habían registrado hasta esa fecha 1.680 pacientes que han consultado y que requieren tratamiento, desconociéndose la prevalencia de pacientes con signos más leves de enfermedad ${ }^{1}$. El diagnóstico se realiza en base a la medición de la actividad del factor VIII en el plasma, calculando el porcentaje respecto de valores establecidos como normales (50-150\%), de acuerdo al cual la enfermedad se clasifica en severa $(<1 \%)$, moderada $(1-5 \%)$ y leve $(6-30 \%)^{2}$. Las mujeres portadoras pueden tener un amplio espectro de niveles de actividad del factor VIII, por lo que en muchos casos no es posible identificarlas por el fenotipo. En algunas de ellas los 
niveles de factor VIII pueden llegar a similares a los que presentan hombres diagnosticados con hemofilia A leve, por lo que tendrían mayor riesgo de sangrado ${ }^{3}$.

Esta enfermedad es causada por alteraciones en el gen del factor VIII (F8), ubicado en el brazo largo del cromosoma X, que comprende $186 \mathrm{~kb}, 26$ exones y 25 intrones, algunos de ellos muy grandes como el intrón 22 (Figura 1$)^{4}$. Este gen tiene dos copias intragénicas ( $F 8 A$ y F8B) dentro del intrón 22 y dos copias extragénicas del gen $F 8 A$ en la región telomérica que son altamente homólogas ${ }^{5}$.En alrededor de $40 \%$ de los pacientes con hemofilia A severa, la alteración genética se debe a una inversión cromosómica entre la región homóloga en el intrón 22 y una de las copias extragénicas del gen F8A. Producto de esta recombinación homóloga, se produce la traslocación de los exones 1 al 22 a la región cercana al telómero en forma invertida, lo que da origen a ausencia completa de expresión del factor VIII y un fenotipo severo. Más recientemente, se ha descrito una inversión similar que involucra también dos regiones homólogas, una en el intrón 1 y otra fuera del gen del factor VIII, que da cuenta de, aproximadamente, $5 \%$ de los pacientes con hemofilia A severa ${ }^{6}$. Deleciones e inserciones, así como mutaciones puntuales de sentido erróneo que generan un codón de término, son responsables del resto de las alteraciones en pacientes con hemofilia A severa. Mutaciones sin sentido, en cambio, se han descrito sobre todo en pacientes con hemofilia A moderada y leve $e^{7}$. Actualmente, se han identificado más de 900 mutaciones distribuidas a lo largo de todo el gen, descritas en la base de datos de hemofilia A HAMSTeRS (http://europium.csc.mrc.ac.uk).

El tamaño del gen, la variedad de mutaciones y la distribución de ellas, hacen que el diagnóstico genético-molecular de pacientes con hemofilia A sea complejo. En nuestro laboratorio sólo se realizaba estudio de las inversiones cromosómicas utilizando el método Southern blot, por lo que no era posible realizar el diagnóstico molecular en el resto de los pacientes con la forma severa, así como en los pacientes con hemofilia A moderada y leve. Para mejorar la sensibilidad diagnóstica también se evaluó la utilidad el análisis indirecto. Este análisis utiliza marcadores genéticos polimórficos, que pueden estar dentro o fuera del gen y que se heredan con la enfermedad, con el fin de identificar a los miembros de la familia que presentan la mutación. Aunque este análisis permite ampliar el diagnóstico molecular a todos los pacientes con hemofilia, tiene la desventaja de ser indirecto, requiere del análisis del caso índice y de varios miembros de la familia afectados, y además no siempre es posible encontrar marcadores informativos, es decir, que sean polimórficos y se hereden con la enfermedad. Tomando en cuenta estas desventajas y que en nuestra experiencia se encontraron marcadores informativos en sólo $66 \%$ de los casos (datos no publicados), no se adoptó este método para su uso como test genético para el estudio de hemofilia A.

En la actualidad, están disponibles metodologías como el PCR para fragmentos largos para el análisis de inversiones cromosómicas ${ }^{8}$, método menos laborioso y más económico, y la secuenciación para el análisis directo de mutaciones puntuales, y de pequeñas deleciones e inserciones. Con estas metodologías es posible identificar la alteración molecular directamente en la gran mayoría de los pacientes con hemofilia A severa, moderada y leve. Esto no sólo permite confirmar el

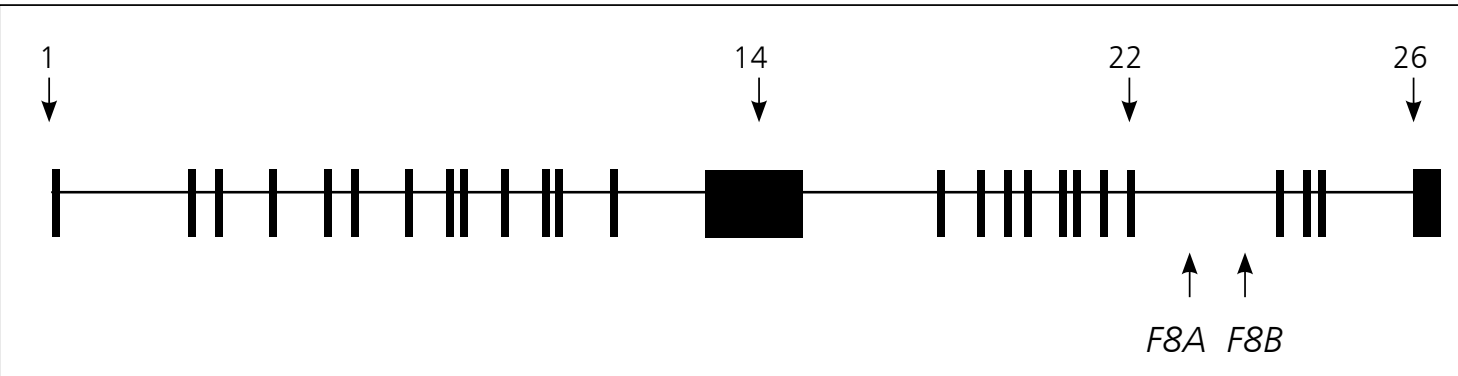

Figura 1. Esquema del gen que codifica para el factor VIII (F8). Arriba: posición de algunos exones, abajo: posición de las copias F8A y F8B dentro del intrón 22, las otras 2 copias de F8A se encuentran fuera del gen a 500 Kb hacia el telómero. 
diagnóstico, sino que también es útil en el manejo clínico, ya que el tipo de mutación permite predecir la severidad de la enfermedad, en especial en los casos esporádicos o sin antecedentes familiares que corresponden a alrededor de un tercio de los pacientes. El tipo de mutación también representa el principal factor de riesgo para desarrollar inhibidores en pacientes en terapia de reemplazo con factor VIII ${ }^{9}$. La identificación de portadoras es una de las aplicaciones más importantes del análisis del gen $F 8$; primero que nada porque es importante para el consejo genético de las portadoras, pero también porque algunas pueden tener niveles normales de actividad del factor VIII y no ser detectadas ${ }^{10}$.

En este trabajo mostramos la implementación y validación de un PCR para el análisis de las inversiones cromosómicas del intrón 22 y del intrón $1, y$ de un método de secuenciación de las regiones codificantes del gen $F 8$, así como el estudio de un grupo de pacientes chilenos con hemofilia A y de posibles portadoras en esas familias.

\section{Pacientes y Método}

\section{Pacientes}

Para la implementación y validación del PCR para fragmentos grandes, se analizaron 4 muestras de pacientes con inversión del intrón 22 previamente estudiadas por Southern blot en nuestro laboratorio y 2 muestras con inversión del intrón 1 estudiadas previamente por PCR en un centro externo. En el caso de la secuenciación, primero se estandarizó la técnica en base al estudio de un individuo de sexo masculino, chileno y sin hemofilia A, y para la validación se analizaron 5 muestras, 4 de pacientes y 1 de una mujer portadora, con mutaciones puntuales previamente identificadas por secuenciación en un centro externo.
Luego, se estudiaron 10 pacientes chilenos con hemofilia A sin estudio genético-molecular previo, no relacionados entre sí, y 12 posibles portadoras relacionadas con 4 de los pacientes. Para la selección y clasificación de los pacientes se consideraron la historia clínica, el patrón de herencia y los datos fenotípicos de laboratorio, basados en la actividad de factor VIII. Un paciente con actividad $<1 \%$ fue catalogado como hemofílico severo, y los 9 restantes como pacientes con hemofilia A leve, ya que presentaron entre 10 y $25 \%$ de actividad de factor VIII. Todos los pacientes firmaron un consentimiento informado, aprobado por el Comité de Ética de la Facultad de Medicina, Pontificia Universidad Católica de Chile.

De todos los sujetos se extrajo ADN de sangre periférica por un método estándar de salting-out. En todos los pacientes se realizó el análisis del intrón 22 y del 1 por PCR de fragmentos grandes. En 5 de los 9 pacientes con hemofilia A leve se estudió el gen completo por secuenciación y en los otros 4 se estudió dirigidamente el exón 23. En las portadoras se buscó la mutación encontrada en el caso índice por secuenciación del exón correspondiente.

\section{PCR para la inversión del intrón 22 e intrón 1}

Para detectar la inversión en el intrón 22, se utilizó un PCR para fragmentos largos descrito previamente ${ }^{9}$, en el que se combinan los partidores $\mathrm{P}, \mathrm{Q}, \mathrm{A}$ y B (Tabla 1). Con estas combinaciones, se amplifican las regiones intragénica y extragénica no alteradas (tubo 1 y 2), así como los genes híbridos que resultan de la recombinación (tubo 3 y 4 ). Un sujeto (mujer u hombre) sin inversión presenta sólo banda en los tubos 1 y 2 dada la presencia de las copias intragénicas y extragénicas no recombinadas. Los pacientes con inversión presentan amplificación en el tubo 2 por la presencia de una

Tabla 1. Esquema utilizado para la detección de la inversión del intrón 22 en 4 reacciones de PCR, combinando los partidores $P$, Q, A y B

\begin{tabular}{|cclcl|}
\hline Tubo & Partidores & \multicolumn{1}{c}{ Producto de } & $\begin{array}{c}\text { Tamaño del } \\
\text { fragmento }\end{array}$ & Presente en \\
\hline 1 & $\mathrm{P}+\mathrm{Q}$ & copia intragénica & $12 \mathrm{~kb}$ & sujetos sin inversión y portadoras \\
\hline 2 & $\mathrm{~A}+\mathrm{B}$ & copia extragénica & $9,8 \mathrm{~kb}$ & sujetos con y sin inversión \\
\hline 3 & $\mathrm{P}+\mathrm{B}$ & genes híbridos por la inversión & $11 \mathrm{~kb}$ & hombres con inversión y portadoras \\
\hline 4 & $\mathrm{~A}+\mathrm{Q}$ & gen & $10,8 \mathrm{~kb}$ & \\
\hline
\end{tabular}


copia extragénica no recombinada, así como en los tubos 3 y 4 por los genes híbridos producto de la recombinación con la otra copia extragénica. En este caso no hay producto en el tubo 1 porque la región intragénica ya no está intacta. Una mujer portadora presenta amplificación en todos los tubos, producto de las regiones no recombinadas de su cromosoma X normal y de los genes híbridos de su cromosoma con inversión del intrón 22.

Para el PCR se utilizó polimerasa para amplificación de fragmentos largos (TaKaRa LA Taq ${ }^{\mathrm{TM}}$, TAKARA Bio Inc), dimetilsulfóxido, 7-deazadGTP, así como el programa recomendado por los autores. Para la detección del producto se realizó electroforesis en gel de agarosa al 0,6\% durante 5 horas a 80 volts, utilizando estándar de peso molecular de 0,125 a $23 \mathrm{~kb}$ (1 DNA/Hind III Fragments, Invitrogen).

En el caso de la inversión en el intrón 1, se realizaron dos reacciones de PCR con reactivos y condiciones similares a los utilizados en el caso de la inversión en el intrón 22. La electroforesis se realizó en agarosa al 1\% por 40 minutos a 130 volts, utilizando un estándar de peso molecular de $1 \mathrm{~kb}$ (1kb DNA Ladder, Fermentas).

\section{Secuenciación del gen del factor VIII (F8)}

Para la amplificación del gen $F 8$ se utilizó un sistema comercial (VariantSEQr ${ }^{\mathrm{TM}}$ Resequencing System, Applied Biosystems) que utiliza partidores a los cuales están acopladas secuencias del fago M13. En el caso del gen $F 8$ son 42 parejas de partidores, que cubren la secuencia codificante de los 26 exones y las uniones exón-intrón. Los productos se purificaron con un sistema comercial (ExoSAP-IT ${ }^{\circledR}$, USB, Affimetrix). La secuenciación se realizó en ambos sentidos con reactivos comerciales (BigDye ${ }^{\circledR}$ Terminator v3.1, Applied Biosystems) y los partidores M13-F y M13-R 3,2 mM. Los productos de la reacción de secuenciación se purificaron con columnas (Performa ${ }^{\circledR}$ DTR, EdgeBio) y se analizaron en el equipo de electroforesis capilar ABI PRISM 3130 (Applied Biosystems).

Para el análisis de las secuencias se utilizó el programa Sequencher v4.5 (Genecodes) y la secuencia de referencia del gen F8 GenBank NG_005114 (publicada en Agosto del 2007). La nomenclatura para designar los cambios se basa en la recomendada en la base de datos de hemofilia A HAMSTeRS (http://hadb.org.uk) y la Human Genome Variation Society, por lo que se restan los 19 aminoácidos del péptido señal, de manera que el número de aminoácido corresponde a la proteína precursora madura.

\section{Resultados}

\section{PCR para la detección de la inversión \\ del intrón 22 y 1}

En el caso de la inversión del intrón 22 sólo se logró obtener amplificación de todos los fragmentos al realizar las reacciones de PCR por separado (Figura 2 izquierda y 2 derecha). En el paciente con hemofilia A severa se detectó la presencia de la inversión del intrón 22, no así en resto de los pacientes.

En el caso de la inversión del intrón 1 fue necesario utilizar concentraciones más altas de los partidores para los fragmentos más grandes $(0,5$ $\mathrm{mM}$ ), disminuyendo simultáneamente las de los más pequeños a $0,25 \mathrm{mM}$, para lograr la amplificación de los tamaños más grandes. En este caso sí fue posible implementar un PCR múltiple, ya que los tamaños de los fragmentos a amplificar eran más pequeños. Ninguno de los pacientes fue positivo para la inversión del intrón 1 , únicamente el control positivo utilizado para la validación (datos no mostrados).

\section{Secuenciación del gen F8}

Las mutaciones y polimorfismos encontrados en las muestras utilizadas para validar el método concordaron con los previamente establecidos, tanto en los pacientes como en la mujer portadora heterocigota (Tabla 2). Con esto se valida la metodología de secuenciación en sus aspectos técnicos, y también el proceso de identificar y nombrar la mutación.

En los 5 pacientes chilenos con hemofilia A leve en que se secuenció la región codificante completa del gen, se encontraron 3 mutaciones previamente descritas en las bases de datos disponibles (Tabla 3). En 3 pacientes se detectó la mutación c.6532C $>\mathrm{T}$ en el exón 23, por lo que se estudiaron dirigidamente otros 4 pacientes con hemofilia A leve, encontrándose en 3 de ellos.

De las 12 posibles portadoras relacionadas a 4 pacientes, 8 mujeres resultaron ser portadoras de la mutación identificada en el caso índice. En un caso, se presentó al estudio sólo una posible portadora en la que no se detectó la mutación del 

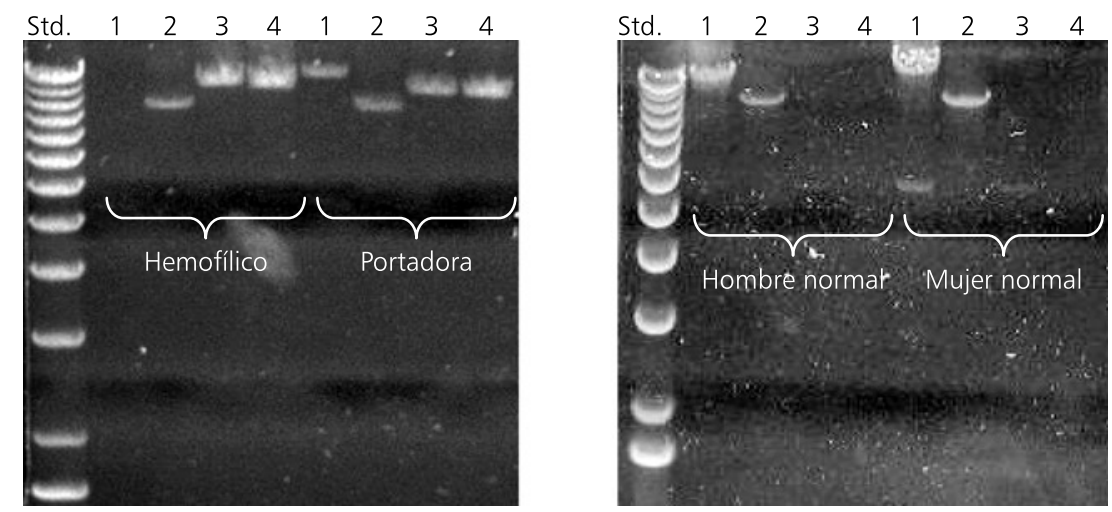

Figura 2. Detección de la inversión del intrón 22 por PCR de fragmentos largos. Std: Estándar de peso molecular (bandas superiores 23130, 9416 y 6557 bp), carriles 1: copia intragénica, carriles 2: copia extragénica, carriles 3 y 4: genes híbridos. Izquierda: paciente con hemofilia A (sin copia intragénica) presenta 1 fragmento A+B de la copia extragénica y 2 fragmentos $\mathrm{P}+\mathrm{B}$ y $\mathrm{A}+\mathrm{Q}$ por los genes híbridos; mujer portadora con los 4 fragmentos. Derecha: hombre y mujer normales con 2 fragmentos $\mathrm{P}+\mathrm{Q}$ de la copia intragénica y $\mathrm{A}+\mathrm{B}$ de la extragénica, no recombinadas.

Tabla 2. Mutaciones y polimorfismos en el gen $F 8$ encontradas en las muestras utilizadas para validar

\begin{tabular}{|ccc|}
\hline Control & Ubicación & Mutación \\
1 & Exón 14 & c.3624delT \\
2 & Exón 7,14 & c.902G $>$ A + c.3780C > G \\
3 & Exón 14 & c.3637delA \\
4 & Intrón 5 & IVS5 + 5G $>$ A \\
5 & Exón 8, 14 & c.1090G $>$ A + c.3780C > G \\
\hline
\end{tabular}

Controles 1, 2, 3 y 4: pacientes hombres; control 5: mujer portadora

caso índice. En las otras 3 familias, se identificaron como portadoras 3 de 4,4 de 4 y 1 de 3 posibles portadoras.

\section{Discusión}

En este trabajo se describe la implementación y validación de metodologías para el estudio genético-molecular de pacientes con hemofilia A y se muestran resultados preliminares en pacientes chilenos. El estudio de las inversiones cromosómicas por PCR de fragmentos largos arrojó resultados concordantes en todas las muestras analizadas

\section{Tabla 3. Mutaciones encontrados en pacientes chilenos con hemofilia $A$ leve en que se secuenció el gen $F 8$}

\begin{tabular}{|ccc|}
\hline Paciente & Ubicación & Mutación \\
\hline HA-1 & Exón 23 & c. $6532 \mathrm{C}>\mathrm{T}$ \\
\hline HA-3 & Exón 23 & c.6532C $>$ T \\
HA-4 & Intrón 3 & IVS9-C $>$ T \\
HA-5 & Exón 19 & c.6047G $>$ A \\
HA-6 & Exón 23 & c.6532C $>$ T \\
\hline
\end{tabular}

previamente, lo que permitió su validación. Este método es sencillo de realizar y de fácil interpretación, sobre todo en comparación con el que se utilizaba con anterioridad (Southern blot), además de reducir el tiempo de análisis y de entrega de resultados, por lo que también sería importante su aplicación a otros estudios como el de síndrome de X-frágil y distrofia miotónica. Esta misma metodología se utilizó para el análisis de la inversión del intrón 1 , pero no se encontraron pacientes con esta alteración en los pacientes estudiados, ya que la mayoría padecían hemofilia A leve. Sin embargo, es probable que no se encuentre con frecuencia en población chilena, ya que la inversión del intrón 
1 se ha descrito como poco frecuente en otras poblaciones, e incluso hay poblaciones como la mexicana en la que no se ha observado ${ }^{11}$.

Un aspecto importante de este trabajo es la utilización de la secuenciación para el análisis de todas las zonas codificantes de un gen tan grande como el del F8 en Chile. Dado el tamaño de este gen de 26 exones, se opta en muchas ocasiones por iniciar la búsqueda de mutaciones puntuales o pequeñas inserciones o deleciones con métodos de screening, como por ejemplo SSCP (Single Strand Conformation Polymorphism). Sin embargo, estos métodos están habitualmente sólo disponibles en laboratorios de investigación, ya que son difíciles de estandarizar y presentan sensibilidades variables de alrededor de $85 \%{ }^{12}$. La secuenciación es el método más sensible para la identificación de este tipo de alteraciones, y dado que los costos han disminuido notablemente, se ha convertido en un método que está cada vez más disponible para el análisis de rutina de esta y otras enfermedades ${ }^{13}$. Este método, en el que se amplifica y secuencia el gen por segmentos, permite además detectar deleciones que pueden abarcar desde un exón hasta el gen completo. Se ha descrito en otras poblaciones que estas alteraciones se presentan en alrededor de $5 \%$ de los pacientes con hemofilia A severa ${ }^{14}$, por lo que su estudio podría contribuir a mejorar la sensibilidad diagnóstica también en población chilena. Otro aspecto positivo de la metodología utilizada, es el uso de la secuencia M13 acoplada a los partidores, ya que simplifica el proceso de secuenciación, pudiéndose realizar en forma simultánea con otros estudios genéticos que también tengan este diseño, además de que permite mejorar la secuencia en los extremos cercanos al partidor.

Cuando la búsqueda de mutaciones no es dirigida, es recomendable cubrir todos los exones y zonas de unión exón-intrón con dos secuenciaciones, de manera que si en una de ellas la secuencia no es clara, se corrobora con la otra para evaluar si hay diferencias con la secuencia de referencia. Este procedimiento garantiza la detección de cambios nucleotídicos por mutaciones en el ADN del paciente y evita falsos positivos por artefactos inducidos por el PCR. En algunas de las regiones cubiertas por los partidores incluidos por el sistema de secuenciación utilizado, se observó una cobertura por más de dos secuencias, por lo que creemos que es posible realizar $15 \%$ menos de reacciones de secuenciación. Tomando en cuenta esto y que todas las mutaciones previamente estudiadas fueron debidamente identificadas con este sistema, no sólo se logra el objetivo de la validación del método, sino que también fue posible optimizar la estrategia y disminuir los costos, sin mermar la solidez del estudio.

Las mutaciones encontradas en estos pacientes chilenos se encuentran todas reportadas previamente en la base de datos de hemofilia A (HAMSTeRS). Lo que más llama la atención de los resultados, es que 6 de $9(66,7 \%)$ de los pacientes con hemofilia A leve presentaron la misma mutación en el exón 23. Esta mutación, que afecta la interacción con el factor von Willebrand y los fosfolípidos, ha sido reportada en el sitio HAMSTeRS en pacientes con hemofilia A moderada a leve en distintas poblaciones. La alta frecuencia observada en el presente trabajo se podría deber a un efecto fundador como se ha observado en hemofilia A en otras poblaciones ${ }^{15} \mathrm{y}$ en otras enfermedades genéticas en nuestro país ${ }^{16}$, lo que se podría confirmar estudiando un mayor número de pacientes y marcadores genéticos que permitan establecer el haplotipo.

El costo, aun cuando ha disminuido, es un aspecto a considerar al realizar secuenciación para el estudio de genes tan grandes como el gen $F 8$, en los que las mutaciones se pueden encontrar a través de todo el gen. Una alternativa que puede ayudar a optimizar los costos, es realizar el análisis por etapas de acuerdo a la frecuencia con que se presenten las alteraciones en cada población y también al fenotipo, tal como se ha propuesto en otros trabajos ${ }^{17}$. De esta manera, en pacientes con hemofilia A severa se podría comenzar con el estudio de la inversión del intrón 22 y del intrón 1 , y continuar con la secuenciación del exón 14, en el que, de acuerdo a lo reportado en el sitio HAMSTeRS, se encuentran con frecuencia mutaciones en pacientes con este fenotipo. En pacientes chilenos con hemofilia A moderada y leve, el análisis podría comenzar con la mutación en el exón 23. De acuerdo a la literatura, se podrían estudiar en un comienzo también los exones 11, comienzo del 14, y continuar luego los exones 4, 7, 9, 16 y resto del exón $14^{18}$. Si con estos estudios no se ha logrado identificar la causa genética, se deberá analizar el resto de los exones. Una propuesta de la secuencia a seguir para el estudio de pacientes chilenos se observa en el diagrama de flujo en la Figura 3. 


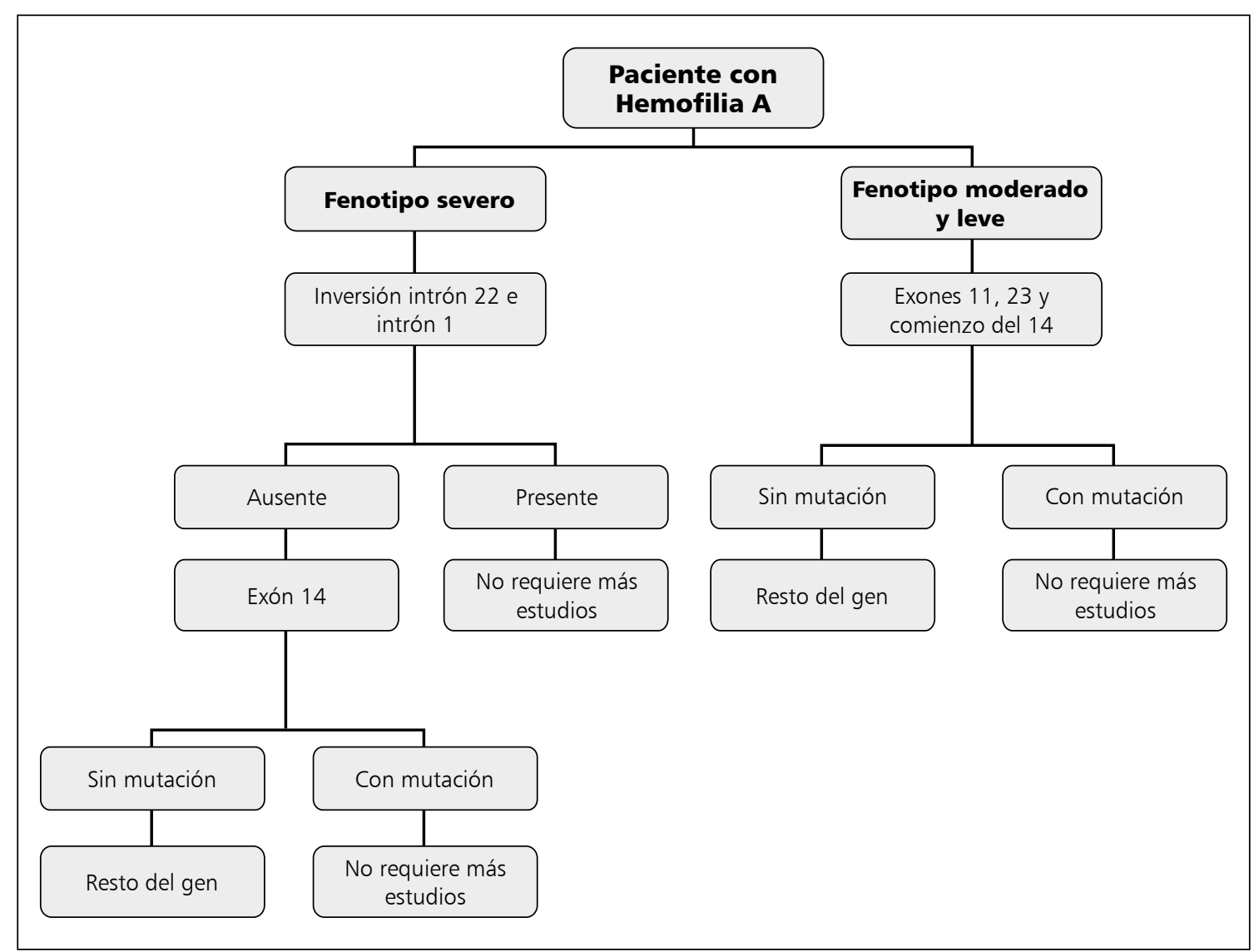

Figura 3. Propuesta de diagrama de flujo para el estudio del gen F8 en pacientes chilenos de acuerdo al fenotipo y la frecuencia con que se observan mutaciones en los distintos exones.

La identificación de portadoras en este estudio permitió identificar a 8 mujeres como portadoras de una mutación que causa hemofilia A, entre sólo 12 mujeres que se sometieron al análisis, lo que muestra que ésta es una de las aplicaciones de mayor implicancia del estudio genético-molecular del gen F8.

De esta forma está disponible en nuestro país, el estudio de las inversiones del intrón 22 y del intrón 1, así como de mutaciones puntuales o inserciones/deleciones por secuenciación del gen F8. Con esto no sólo es posible confirmar el diagnóstico y contribuir al manejo clínico del paciente en base al tipo de mutación, sino que también permite, una vez que se identifica la mutación en el caso índice, estudiar a los familiares dirigidamente, ya sean posibles portadoras como otros posibles afectados, y otorgar mejor consejo genético.

\section{Referencias}

1. Ministerio de Salud. Guía Clínica Hemofilia. Santiago: Minsal, 2007.

2. White GC 2nd, Rosendaal F, Aledort LM, Lusher JM, Rothschild C, Ingerslev J. Factor VIII and Factor IX Subcommittee. Definitions in hemophilia. Recommendation of the scientific subcommittee on factor VIII and factor IX of the scientific and standardization committee of the International Society on Thrombosis and Haemostasis. Thromb Haemost 2001; 85: 560.

3. Plug I, Mauser-Bunschoten EP, Bröcker-Vriends AH, Van Amstel HK, Van DerR Bom JG, Van Diemen-Homan JE et al. Bleeding in carriers of hemophilia. Blood 2006; 108: 52-6.

4. Bolton-Maggs PH, Pasi KJ. Haemophilias A and B. Lancet 2003; 361: 1801-9.

5. Graw J, Brackmann HH, Oldenburg J, Schneppenheim 
R, Spannagl M, Schwaab R. Haemophilia A: from mutation analysis to new therapies. Nat Rev Genet 2005; 6: 488-501.

6. Bagnall RD, Waseem N, Green PM, Giannelli F. Recurrent inversion breaking intron 1 of the factor VIII gene is a frequent cause of severe hemophilia A. Blood 2002; 99: 168-74.

7. Brower CL, Thompson AR (última revisión 2008). Hemophilia A. In: GeneReviews at GeneTests (http://www. genetests.org) último acceso 22-02-2010.

8. Liu Q, Nozari G, Sommer SS. Single-tube polymerase chain reaction for rapid diagnosis of the inversion hotspot of mutation in hemophilia A. Blood 1998; 92: 1458-9.

9. Dasgupta S, Navarrete AM, Delignat S, Wootla B, Andre S, Nagaraja V, et al. Immune response against therapeutic factor VIII in hemophilia A patients-a survey of probable risk factors. Immunol Lett 2007; 110: 23-8.

10. Pruthi RK. Hemophilia: a practical approach to genetic testing. Mayo Clin Proc 2005; 80: 1485-99.

11. Mantilla-Capacho JM, Beltrán-Miranda CP, Luna-Záizar H, Aguilar-López L, Esparza-Flores MA, López-Guido B, et al. Frequency of intron 1 and 22 inversions of Factor VIII gene in Mexican patients with severe hemophilia A. Am J Hematol 2007; 82: 283-7.
12. Castaldo G, D’Argenio V, Nardiello P, Zarrilli F, Sanna V, Rocino A, et al. Haemophilia A: molecular insights. Clin Chem Lab Med 2007; 45: 450-61.

13. Santacroce R, Acquila M, Belvini D, Castaldo G, Garagiola I, Giacomelli SH, et al. Identification of 217 unreported mutations in the F8 gene in a group of 1,410 unselected Italian patients with hemophilia A. J Hum Genet 2008; 53: 275-84.

14. Becker J, Schwaab R, Möller-Taube A, Schwaab U, Schmidt W, Brackmann HH, et al. Characterization of the factor VIII defect in 147 patients with sporadic hemophilia A: family studies indicate a mutation typedependent sex ratio of mutation frequencies. Am J Hum Genet 1996; 58: 657-70.

15. Acquila M, Pasino M, Lanza T, Bottini F, Molinari AC, Bicocchi MP. Duplication of exon 13 causes one third of the cases of mild hemophilia A in northern Italy. Haematologica 2004; 89: 758-9.

16. Molina G, González FJ, Cave R, Cornejo de M, Navarro $\mathrm{S}$, Deglin M, et al. Estudio clínico-genético molecular de la fibrosis quística en la V Región, Chile. Rev Med Chile 2002; 130: 850-8.

17. Bogdanova N, Markoff A, Eisert R, Wermes C, Pollmann $\mathrm{H}$, Todorova $\mathrm{A}$ et al. Spectrum of molecular defects and mutation detection rate in patients with mild and moderate hemophilia A. Hum Mutat 2007; 28: 54-60. 